\title{
Higher CSF sTREM2 attenuates ApoE4- related risk for cognitive decline and neurodegeneration
}

Nicolai Franzmeier ${ }^{1 *}$ (D, M. Suárez-Calvet ${ }^{2,3,4}$, Lukas Frontzkowski ${ }^{1}$, Annah Moore ${ }^{5}$, Timothy J. Hohman ${ }^{5}$, Estrella Morenas-Rodriguez ${ }^{6}$, Brigitte Nuscher ${ }^{6}$, Leslie Shaw ${ }^{7}$, John Q. Trojanowski ${ }^{8}$, Martin Dichgans ${ }^{1,9,10}$, Gernot Kleinberger ${ }^{11}$, Christian Haass ${ }^{6,9,10}$, Michael Ewers ${ }^{1,10^{*}}$ and for the Alzheimer's Disease Neuroimaging Initiative (ADNI)

\begin{abstract}
Background: The Apolipoprotein E $\varepsilon 4$ allele (i.e. ApoE4) is the strongest genetic risk factor for sporadic Alzheimer's disease (AD). TREM2 (i.e. Triggering receptor expressed on myeloid cells 2) is a microglial transmembrane protein brain that plays a central role in microglia activation in response to AD brain pathologies. Whether higher TREM2related microglia activity modulates the risk to develop clinical AD is an open question. Thus, the aim of the current study was to assess whether higher sTREM2 attenuates the effects of ApoE4-effects on future cognitive decline and neurodegeneration.

Methods: We included 708 subjects ranging from cognitively normal (CN, $n=221)$ to mild cognitive impairment $(\mathrm{MCl}, n=414)$ and AD dementia $(n=73)$ from the Alzheimer's disease Neuroimaging Initiative. We used linear regression to test the interaction between ApoE4-carriage by CSF-assessed STREM2 levels as a predictor of longitudinally assessed cognitive decline and MRI-assessed changes in hippocampal volume changes (mean followup of 4 years, range of 1.7-7 years).

Results: Across the entire sample, we found that higher CSF sTREM2 at baseline was associated with attenuated effects of ApoE4-carriage (i.e. sTREM $2 \times$ ApoE4 interaction) on longitudinal global cognitive $\left(p=0.001\right.$, Cohen's $f^{2}=$ $0.137)$ and memory decline $\left(p=0.006\right.$, Cohen's $\left.f^{2}=0.104\right)$ as well as longitudinally assessed hippocampal atrophy $\left(p=0.046\right.$, Cohen's $\left.f^{2}=0.089\right)$, independent of CSF markers of primary AD pathology (i.e. $A \beta_{1-42}, p$-tau 181$)$. While overall effects of sTREM2 were small, exploratory subanalyses stratified by diagnostic groups showed that beneficial effects of sTREM2 were pronounced in the MCl group.

Conclusion: Our results suggest that a higher CSF sTREM2 levels are associated with attenuated ApoE4-related risk for future cognitive decline and AD-typical neurodegeneration. These findings provide further evidence that TREM 2 may be protective against the development of AD.
\end{abstract}

Keywords: Alzheimer's disease, ApoE4, Microglial activation, sTREM2, Cognitive decline, Neurodegeneration

\footnotetext{
* Correspondence: Nicolai.franzmeier@med.uni-muenchen.de;

Michael.ewers@med.uni-muenchen.de

${ }^{1}$ Institute for Stroke and Dementia Research (ISD), University Hospital, Ludwig

Maximilian University (LMU), Munich, Germany

Full list of author information is available at the end of the article
}

\section{$\triangle B M C$}

(c) The Author(s). 2020 Open Access This article is licensed under a Creative Commons Attribution 4.0 International License, which permits use, sharing, adaptation, distribution and reproduction in any medium or format, as long as you give appropriate credit to the original author(s) and the source, provide a link to the Creative Commons licence, and indicate if changes were made. The images or other third party material in this article are included in the article's Creative Commons licence, unless indicated otherwise in a credit line to the material. If material is not included in the article's Creative Commons licence and your intended use is not permitted by statutory regulation or exceeds the permitted use, you will need to obtain permission directly from the copyright holder. To view a copy of this licence, visit http://creativecommons.org/licenses/by/4.0/ The Creative Commons Public Domain Dedication waiver (http://creativecommons.org/publicdomain/zero/1.0/) applies to the data made available in this article, unless otherwise stated in a credit line to the data. 


\section{Background}

The $A P O E$ \&4 allele (i.e. ApoE4) is the strongest genetic risk factor for sporadic Alzheimer's disease (AD) [1]. ApoE4-homozygotes have a 31-40\% lifetime risk for developing $\mathrm{AD}$ dementia [2] and show 10-year earlier AD symptom onset than ApoE4 non-carriers [3]. ApoE4-carriage is associated with $\mathrm{AD}$-typical alterations in cerebrospinal-fluid (CSF) $A \beta_{1-42}$ [4] and with earlier and stronger PET-assessed amyloid-beta $(\mathrm{A} \beta)[5,6]$ and tau accumulation [7]. Additionally, elderly ApoE4carriers show faster cognitive decline than ApoE4-non carriers [8-11] as well as AD-typical temporo-parietal neurodegeneration [12]. Together, ApoE4-carriers are at increased risk of developing primary AD pathology, cognitive decline and AD dementia [2, 5, 6]. Recent GWAS suggest that the brain's immune response may modulate AD risk [13-15]. This is supported by a 2-4-fold elevated odds ratio for $\mathrm{AD}$ in carriers of loss-of-function risk variants in the TREM2 gene (triggering receptor expressed on myeloid cells 2, i.e. in the brain preferentially expressed on microglia) [16]. The TREM2/DAP12 signaling complex regulates microglial responses to pathogens, enhancing microglia motility, chemotaxis and phagocytosis [17-22]. In APP or Tau transgenic mice, TREM2-mediated microglia activation has been shown to promote $A \beta$ phagocytosis [20], to limit $A \beta$ seeding [22], tau hyperphosphorylation [23] and tau seeding around neuritic plaques [24]. TREM2-deficiency has been associated with memory impairment in tau transgenic mice, while higher TREM2 expression was neuroprotective and beneficial for memory [25]. Similarly, enhancing TREM2 signaling via agonistic antibodies can promote microglial survival and $A \beta$ phagocytosis in $A \beta$ mice [26]. Further supporting protective TREM2 effects in $\mathrm{AD}$, TREM2-deficiency is associated with reduced microglia clustering and increased tau seeding around neuritic plaques [24]. Hence, TREM2-related microglial activation may attenuate downstream consequences of primary $\mathrm{AD}$ pathology and modulate ApoE4-related risk for AD symptoms and neurodegeneration.

Soluble TREM2 (sTREM2) originates from ADAM10/ 17-mediated TREM2-ectodomain shedding and can be detected in the CSF [19, 27-29], hence sTREM2 levels are interpreted as proxies of microglial TREM2 signaling [27-29]. Supporting this, brain sTREM2 levels are highly correlated with TSPO-PET-assessed microglial activation in mice [30] and are decreased in mice carrying the TREM2 p.T66M variant that typically locks microglia in a homeostatic state [31]. We previously found in sporadic and familial AD that CSF sTREM2 levels rise before symptom onset and mostly correlate with increasing CSF tau levels, suggesting an adaptive immune response $[32,33]$. Importantly, higher CSF sTREM2 levels at a given level of primary $\mathrm{AD}$ pathology (measured as the
CSF $A \beta_{1-42}$ and $p$-tau $\left.{ }_{181}\right)$, were associated with attenuated future cognitive decline, neurodegeneration and delayed conversion to $\mathrm{AD}$ dementia in amyloid-positive individuals [34]. These findings suggested that TREM2related microglial activation is associated with attenuated cognitive decline and neurodegeneration in AD, especially when TREM2 levels are high at a given level of tau pathology.

Here, we asked whether CSF sTREM2 attenuates genetic risk for i) cognitive decline and ii) AD-typical neurodegeneration as conferred by the most important genetic $\mathrm{AD}$ risk factor ApoE4. This question is clinically important, since TREM2-signalling is modifiable [26] and may constitute a target for $\mathrm{AD}$ prevention. In a large sample of 708 subjects ranging from cognitively normal to dementia, we thus determined whether higher CSF sTREM2 levels attenuate the association between ApoE4-carriage and future cognitive decline or MRIassessed neurodegeneration.

\section{Methods \\ Sample}

We included 708 subjects from the ADNI database with available ApoE4 genotyping, baseline CSF values of $A \beta_{1-}$ 42, p-tau ${ }_{181}$ and sTREM2 as well as at least 1.5 years of clinical follow-up assessment (i.e. baseline plus two follow-up visits). ApoE4 genotyping methods can be found online (http://adni.loni.usc.edu/methods/). Subjects were characterized as ApoE4 carriers, when carrying at least one ApoE4 allele. Selection bias was tested against the ADNI baseline cohort of 1784 subjects. Here, we found no differences in gender or education between our selected sample and the baseline ADNI cohort, but selected subjects were significantly older $(p<0.05)$ than the entire ADNI cohort. Subjects were clinically classified by ADNI centers as cognitively normal $(\mathrm{CN}$, MMSE $>24, C D R=0$, non-depressed), mild cognitively impaired (MCI; MMSE $>24, \quad \mathrm{CDR}=0.5, \quad$ objective memory-loss on the education adjusted Wechsler Memory Scale II, preserved activities of daily living) [35] or AD dementia following-pre-established criteria [35]. Based on pre-established CSF $\mathrm{A} \beta_{1-42}$ cut-offs at 976.6 $\mathrm{pg} / \mathrm{ml}$, subjects were classified as $\mathrm{A} \beta+$ (i.e. below 976.6 $\mathrm{pg} / \mathrm{ml}$ ) or $\mathrm{A} \beta$ - (i.e. above $976.6 \mathrm{pg} / \mathrm{ml}$ ) [36]. ADNI was ethically approved by the institutional review board of all participating sites, subjects provided written informed consent.

\section{CSF biomarker assessment}

CSF sTREM2 levels were measured using a previously described ELISA approach [19, 27]. Detailed methods of the CSF sTREM2 assessments can be found online in the ADNI LONI Image \& Data Archive (https://ida.loni. usc.edu). CSF $A \beta_{1-42}$ and $p-$ tau $_{181}$ levels were measured 
by the ADNI biomarker core at the University of Pennsylvania, using the electrochemiluminiscence immunoassays Elecsys on a fully automated Elecsys cobas e 601 instrument and a single lot of reagents for each biomarker.

\section{Hippocampal volume and cortical thickness assessment}

Hippocampal volume was assessed longitudinally in 558 of the 708 subjects who had $\geq 3$ available $3 \mathrm{~T}$ MRIassessments provided by the ADNI imaging core at UCSF [37]. Hippocampal volumes were assessed on $3 \mathrm{~T}$ structural MRI (MPRAGE) using established FreeSurfer pipelines (Version 5.1). Protocols of the ADNI FreeSurfer-based pipelines are available online (http:// adni.loni.usc.edu/) and in previous publications [38]. All analyses using hippocampal volumes as a dependent variable were controlled for Freesurfer-assessed intracranial volume.

\section{Cognitive assessment}

Memory performance was determined using ADNIMEM, a composite memory score that summarizes multiple tests including the Rey Auditory Verbal Learning Test, AD Assessment Scale - Cognitive Subscale, Word Recall of the MMSE and the Wechsler Logical Memory Scale II [39]. For global cognition, we used the ADAS13 $[40,41]$, which is frequently used as a primary endpoint in clinical trials. Please note that ADNI-MEM and ADAS13 scores have an inverse relationship, i.e. lower ADNI-MEM and higher ADAS13 scores indicate worse cognitive performance.

\section{Statistical analysis}

Baseline characteristics were compared between diagnostic groups using ANOVAs for continuous and $x^{2}$ tests for categorical measures. To validate ApoE4 as a major $\mathrm{AD}$ risk factor in the current sample, we tested whether ApoE4-carriage is associated with more abnormal CSF AD biomarkers (i.e. CSF $\mathrm{A} \beta_{1-42}$ \& p-tau ${ }_{181}$ ), using ANCOVAs, controlling for age, gender, education and diagnosis. We used equivalent ANCOVA models to assess whether ApoE4-carriage was associated with elevated CSF sTREM2 levels.

Next, we tested whether ApoE4-carriage was associated with faster rates of longitudinally assessed cognitive decline and neurodegeneration and whether higher sTREM2 levels moderated this association. As measures of cognition, we used ADNI-MEM and ADAS13. As a measure of neurodegeneration, we used Freesurferderived hippocampal volumes. To determine annual change rates in cognition and hippocampal volume, we employed a pre-established approach [42] in which we fitted linear mixed models with ADNI-MEM, ADAS13, hippocampal volumes or cortical thickness values as the dependent variable and time (i.e. years from baseline) as the independent variable, controlling for random slope and intercept. From the linear mixed models, we then derived a slope estimate for change in ADAS13, ADNI-MEM or hippocampal volume across time (i.e. change per year) for each subject. For each measure, longitudinal analyses were restricted to subjects with at least 3 available timepoints (i.e. $n=708$ for ADNI-MEM and ADAS13; $n=558$ for hippocampal volume). Using ANCOVAs, we tested whether ApoE4carriage was associated with faster annual change rates in ADNI-MEM, ADAS13, hippocampal volume. These analyses were controlled for age, gender, education, diagnosis, follow-up time, baseline values of the dependent variable and intracranial volume when using hippocampal volume as a dependent variable.

For our main hypothesis, we assessed whether higher sTREM2 attenuates the association between ApoE4carriage and cognitive decline as well as hippocampal volume changes. First, we applied three ANCOVA models, testing the interaction sTREM2 $\mathrm{x}$ ApoE4carriage on annual rates of change in ADNI-MEM, ADAS13 or hippocampal volume. When using ADNIMEM or ADAS13 change rates as dependent variables, models were controlled for main effects of sTREM2, ApoE4, p-tau ${ }_{181}, A \beta_{1-42}$, age, gender, education, diagnosis, follow-up time and baseline cognition (i.e. ADNIMEM or ADAS13). When using hippocampal volume change rates as the dependent variable, the model was controlled for main effects of sTREM2, ApoE4, A $\beta_{1-42}$, $\mathrm{p}$-tau ${ }_{181}$, age, gender, education, diagnosis, follow-up time, as well as baseline hippocampal volume and intracranial volume. These covariates were selected to ensure that ApoE4 x sTREM2 interactions were not driven by baseline differences in primary $\mathrm{AD}$ pathology (i.e. $\mathrm{A} \beta_{1-}$ ${ }_{42}, \mathrm{p}$-tau $\left.{ }_{181}\right)$, sTREM2 or any of the demographic variables. To account for multiple testing in our primary analysis using two different cognitive endpoints (i.e. ADNI-MEM \& ADAS13), we applied a Bonferronicorrected alpha-threshold of 0.025 (i.e. accounting for 2 tests). For all significant ApoE4 x sTREM2 interaction effects, we further computed effect size estimates (i.e. Cohen's $\mathrm{f}^{2}$ ) which are interpreted as follows: $0.1=$ small effect, $0.25=$ medium effect, $0.4=$ large effect. Note, that interaction effects were plotted using scores of the dependent variables that were residualized for CSF $A \beta_{1-}$ 42 and $\mathrm{p}$-tau ${ }_{181}$, in order to illustrate sTREM2 effects on the association between ApoE4 vs. cognitive and hippocampal volume changes independent of primary $\mathrm{AD}$ markers. As additional exploratory analyses, we re-ran the above described linear models this time testing whether a higher sTREM2/p-tau 181 ratio consistently moderated the effect of ApoE4 on cognitive decline and neurodegeneration. Theses exploratory analyses were 
motivated by our previous work, showing that a higher sTREM2/p-tau 181 ratio is associated with delayed conversion to $A D$ dementia in amyloid-positive individuals [34]. All statistical analyses were conducted in R (Version 3.6.1).

\section{Results}

Sample demographics, biomarker and cognitive data are shown in Table 1.

\section{ApoE4-carriage is associated with more abnormal AD biomarkers and faster cognitive decline but not with changed CSF sTREM2 levels}

First, we assessed the association between ApoE4carriage, baseline CSF biomarkers, rates of cognitive decline and hippocampal atrophy. We found an association between ApoE4-carriage and both decreased baseline CSF- $A \beta_{1-42} \quad(F=182.12, \quad p<0.001, \quad$ Cohens $D=0.96$, Fig. 1a, Supplementary Figure 1A for a stratification by diagnosis $)$ and higher baseline $\mathrm{p}$-tau $\mathrm{tan}_{181}$ levels $(\mathrm{F}=110.53$, $p<0.001$, Cohens $\mathrm{D}=0.76$, Fig. 1b, Supplementary Figure $1 \mathrm{~B}$ stratified by diagnosis), using ANCOVAs controlled for age, gender, education and diagnosis. ApoE4carriage was, however, not associated with baseline CSF sTREM2 levels $(\mathrm{F}=1.47, p=0.225$, Cohens $\mathrm{D}=0.03$, Fig. 1c, ANCOVA controlled for age, gender, education and diagnosis, Supplementary Figure $1 \mathrm{C}$ stratified by diagnosis), consistent with previous reports [43]. For cognition, we found an association between ApoE4-carriage and faster decline in both ADNI-MEM $(F(1,693)=64.61$, $p<0.001$, Cohens $\mathrm{D}=0.51$, Fig. 2a) and ADAS13 (F (1, $690)=91.10, p<0.001$, Cohens $\mathrm{D}=0.58$, Fig. $2 b)$ controlling for age, gender, education, diagnosis, follow-up duration and baseline cognition (i.e. ADNI-MEM or ADAS13 respectively). In a similar vein, ApoE4-carriage was associated with faster hippocampal atrophy, controlling for age, gender, education, diagnosis baseline hippocampal and intracranial volume $(\mathrm{F}=12.39, p<0.001$, Cohens $\mathrm{D}=0.30$ ). Together, these results indicate that ApoE4-carriage is - independently of clinical diagnostic status - associated with AD-typical CSF amyloid and tau levels, as well as cognitive decline and neurodegeneration in the current ADNI sample. However, ApoE4carriage is not associated with elevated CSF sTREM2 levels.

\section{ApoE4-effects on cognitive decline are attenuated at higher sTREM2 levels}

We next tested our main hypothesis that higher sTREM2 levels are associated with attenuated effects of ApoE4-carriage on future cognitive decline. For our main analysis, we found a significant interaction between sTREM2 and ApoE4-carriage on the change rates in ADAS13 $\left(\mathrm{F}=12.89, p<0.001\right.$, Cohens $f^{2}=0.137$, Fig. $\left.2 \mathrm{a}\right)$ and ADNI-MEM $\left(\mathrm{F}=7.459, p=0.006\right.$, Cohens $f^{2}=0.104$, Fig. 2b) controlling for main effects of CSF p-tau 181 , $\mathrm{A} \beta_{42}$, sTREM2, ApoE4 plus age, gender, education, diagnosis, follow-up duration, and baseline cognition (i.e. ADNI-MEM or ADAS13). Note that changes in ADAS13 and ADNI-MEM as shown in Fig. 2 have been split at the median and residualized for CSF A $\beta$ and $p$ $\operatorname{tau}_{181}$ for illustrational purposes only, while statistics were derived using continuous and raw ADAS13 or ADNI-MEM scores. As shown in Fig. 2a\&b, higher sTREM2 levels at baseline were associated with an attenuated effect of ApoE4-carriage on future cognitive

Table 1: Sample characteristics

\begin{tabular}{|c|c|c|c|c|}
\hline $\begin{array}{l}\text { ADNI Sample } \\
(N=708)\end{array}$ & $\begin{array}{l}\mathrm{CN} \\
(n=221)\end{array}$ & $\begin{array}{l}\mathrm{MCl} \\
(n=414)\end{array}$ & $\begin{array}{l}\mathrm{AD} \\
(n=73)\end{array}$ & $p$-value \\
\hline Age (M/SD) & $74.25(6.08)^{b}$ & $71.82(7.45)^{\mathrm{a}, \mathrm{c}}$ & $74.17(8.37)^{b}$ & $<0.001$ \\
\hline Gender (male/female)) & 115/106 & $244 / 170$ & $38 / 35$ & 0.190 \\
\hline Education (M/SD) & $16.36(2.73)^{c}$ & $16.14(2.74)^{c}$ & $15.18(3.06)^{a, b}$ & 0.007 \\
\hline Follow-up in years (M/SD) & $4.89(2.5)^{\mathrm{b}, \mathrm{c}}$ & $4.24(1.81)^{\mathrm{a}, \mathrm{c}}$ & $2.15(0.44)^{a, b}$ & $<0.001$ \\
\hline ApoE4-status (pos./neg.) & $55 / 166$ & $203 / 211$ & $60 / 13$ & $<0.001$ \\
\hline ApoE-alleles (22/23/33/24/34/44) & 0/30/136/1/50/4 & 0/28/183/6/148/49 & $0 / 0 / 13 / 2 / 36 / 22$ & $<0.001$ \\
\hline Amyloid-status (pos./neg.) & $72 / 148$ & $245 / 169$ & $73 / 0$ & $<0.001$ \\
\hline CSF-A $\beta_{1-42}(M / S D)$ & $1243.25(421.93)^{b, c}$ & $976.67(444.52)^{\mathrm{a}, \mathrm{c}}$ & $545.03(159.15)=$ & $<0.001$ \\
\hline CSF-p-tau 181 (M/SD) & $21.97(9.24)^{\mathrm{b}, \mathrm{c}}$ & $27.55(14.27)^{a, c}$ & $36.77(14.33)^{a, b}$ & $<0.001$ \\
\hline CSF sTREM2 (M/SD) & $4258.60(2183.71)$ & $4094.56(2104.86)$ & $4370.51(2194.07)$ & 0.465 \\
\hline ADNI-MEM (M/SD) & $1.07(0.58)^{\mathrm{b}, \mathrm{c}}$ & $0.23(0.67)^{a, c}$ & $-0.84(0.50)^{a, b}$ & $<0.001$ \\
\hline ADAS13 (M/SD) & $9.08(4.33)^{\mathrm{b}, \mathrm{c}}$ & $15.80(6.61)^{\mathrm{a}, \mathrm{c}}$ & $28.82(6.88)^{a, b}$ & $<0.001$ \\
\hline MMSE (M/SD) & $29.11(1.14)^{\mathrm{b}, \mathrm{c}}$ & $27.80(1.78)^{\mathrm{a}, \mathrm{c}}$ & $23.12(1.89)^{a, b}$ & $<0.001$ \\
\hline
\end{tabular}

CN Cognitively Normal, MCI Mild Cognitive Impairment, MMSE Mini-Mental State Exam, ADNI-MEM Alzheimer's disease Neuroimaging Initiative - memory composite, ${ }^{a}=$ sig. Different from $\mathrm{CN}^{\mathrm{b}}{ }^{\mathrm{b}}=$ sig. Different from $\mathrm{MCl}^{,}{ }^{\mathrm{c}}=$ sig. Different from Dementia 

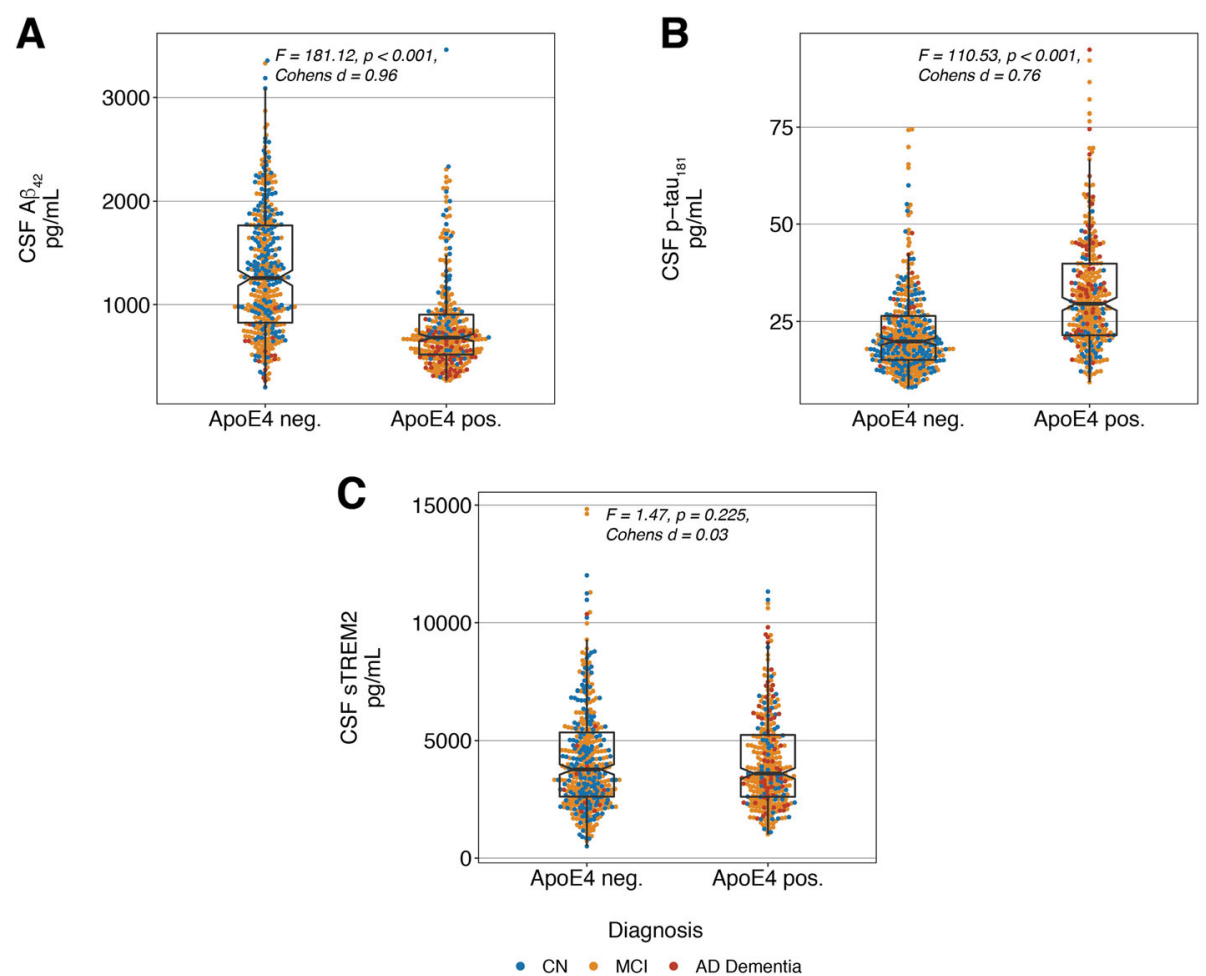

Fig. 1 ApoE4 is associated with abnormal CSF $A \beta_{1-42}$ and $p$-tau ${ }_{181}$ but not with sTREM2. Associations between ApoE4-status, baseline AD biomarkers ( $(\mathbf{a}) A \beta_{1-42},(\mathbf{b}) \mathrm{p}$-tau ${ }_{181}$ ) and (c) baseline sTREM2. F- and $p$-values were determined using ANCOVAs controlling for age, gender, education and diagnosis

decline (for equivalent plots using non-residualized cognitive change scores see supplementary Figure 2). Results remained significant after applying a Bonferronicorrected alpha-threshold of 0.025 , accounting for two independent cognitive endpoints. Congruent interaction effects were found when testing the sTREM2/p-tau $181 \mathrm{x}$ ApoE4 interaction, controlling for main effects of sTREM2, ApoE4, p-tau 181 , age, gender, education, diagnosis, follow-up duration, as well as baseline cognition (ADAS13: $\mathrm{F}=9.39, p=0.002$, Cohens $f^{2}=0.117$; ADNIMEM: $\mathrm{F}=14.54, p<0.001$, Cohens $f^{2}=0.145$ ).

In an exploratory step, we further assessed whether sTREM2 $x$ ApoE4 interactions on cognitive decline were driven by the presence of abnormal amyloid levels. To address this, we additionally restricted the models to $A \beta+$ subjects, where we found congruent results with our main analyses (ADAS13: $\mathrm{F}=8.08, p=0.005$, Cohens $f^{2}=0.147$, Fig. 2d; ADNI-MEM: $\mathrm{F}=5.90, p=0.016$, Cohens $f^{2}=0.125$, Fig. 2e). To further determine whether our findings were driven by clinical diagnosis, we repeated the analyses stratified by diagnostic group and found sTREM $2 \times$ ApoE4 interaction effects to be in line with our main analyses in MCI (ADNI-MEM: F = 5.00, $p=0.026$, Cohens $f^{2}=0.112$; ADAS13: $\mathrm{F}=10.41$, $p=0.001$, Cohens $f^{2}=0.162$ ). In $\mathrm{CN}$ and $\mathrm{AD}$, we found trend level sTREM2 $\mathrm{x}$ ApoE4 interactions for rates of change in ADAS13 (CN: F $=3.78, p=0.053$, Cohens $f^{2}=$ 0.135; $\mathrm{AD}: \mathrm{F}=3.23, p=0.07$, Cohens $f^{2}=0.230$ ), but no effects on rates of change in ADNI-MEM (CN: $p=0.95$, AD: $p=0.22$ ). Collectively, these analyses suggest that higher sTREM2 levels are associated with attenuated effects of ApoE4-carriage on future cognitive decline.

\section{High sTREM2 levels attenuate ApoE4-effects on neurodegeneration}

Lastly, we determined whether higher sTREM2 levels attenuate the effect of ApoE4-carriage on neurodegeneration (i.e. annual hippocampal volume change rates). Here, we found a significant sTREM2 $\mathrm{x}$ ApoE4 interaction on annual hippocampal volume changes $(\mathrm{F}=4.00$, $p=0.046$, Cohens $f^{2}=0.089$, Fig. 2c, model controlled for main effects of ApoE4 and sTREM2, baseline levels of $\mathrm{p}-\mathrm{tau}_{181}, \mathrm{~A} \beta_{1-42}$, age, gender, education, diagnosis, follow-up duration, baseline hippocampal and intracranial volume). Figure $2 \mathrm{c}$ illustrates that ApoE4-carriers with higher sTREM2 levels at baseline showed slower future hippocampal atrophy. The sTREM2 x ApoE4 interaction remained significant when tested in $\mathrm{MCI}$ $\left(\mathrm{F}=7.72, p=0.006\right.$ Cohens $\left.f^{2}=0.147\right)$, but was nonsignificant when tested in $\mathrm{CN}(p=0.35), \mathrm{AD}(p=0.20)$ or $\mathrm{A} \beta+$ only $(\mathrm{F}=2.43, p=0.120$, Fig. $2 \mathrm{f})$. A congruent effect on hippocampal change rates was found when 


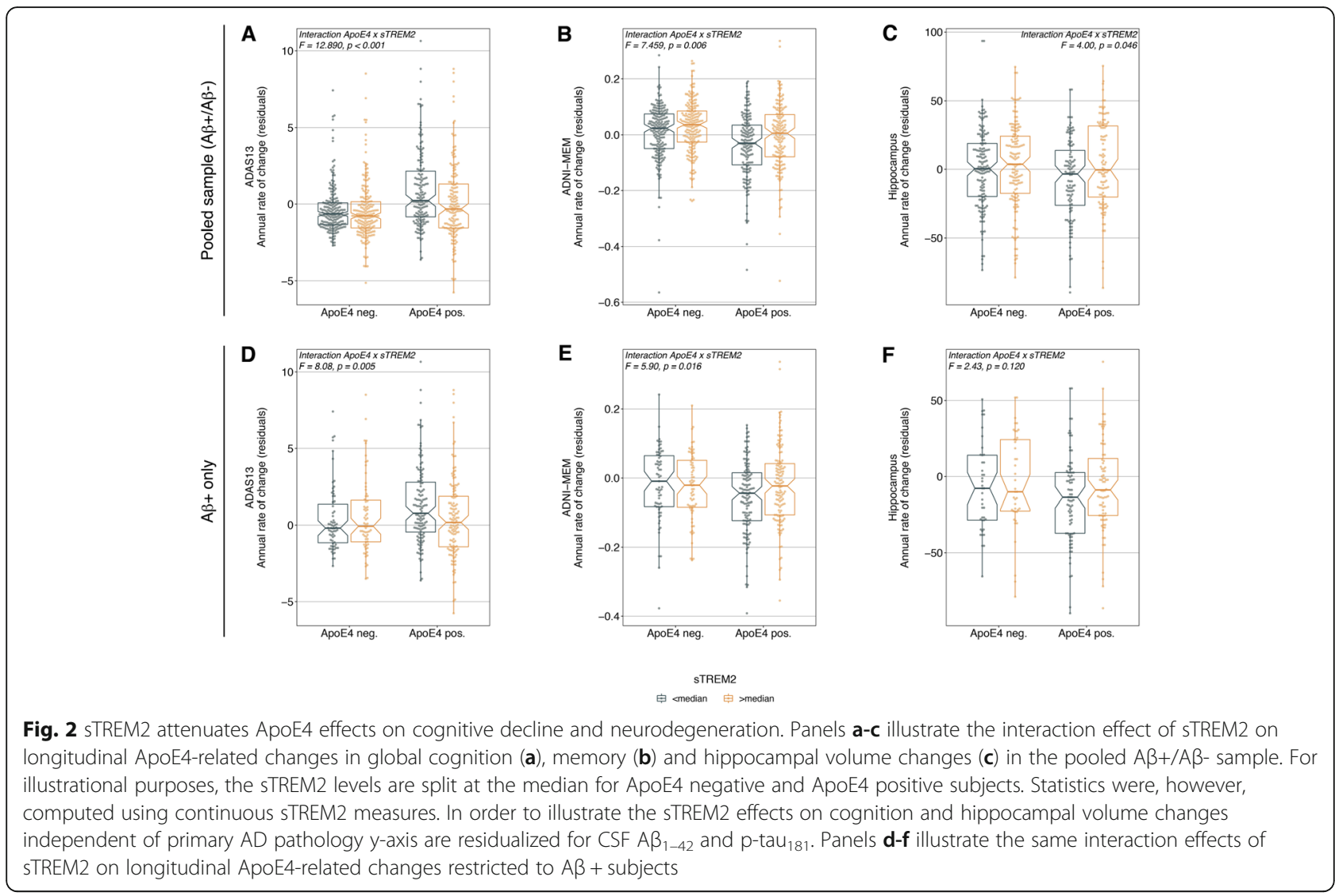

testing the sTREM2/p-tau $181 \times$ ApoE4 interaction $(\mathrm{F}=$ 9.78, $p=0.002$, Cohens $f^{2}=0.134$, i.e. using the same covariates as the previous model) in the entire $A \beta+/ A \beta-$ sample. Faster hippocampal atrophy rates were associated with faster decline in ADNI-MEM $(\beta=0.34$, $\mathrm{T}=8.37, p<0.001)$ and ADAS13 $(\beta=-0.21, \mathrm{~T}=-5.32$, $p<0.001)$, as shown by linear regression controlling for age, gender, education and diagnosis. Together, these findings indicate that higher sTREM2 levels are associated with slower hippocampal atrophy in ApoE4carriers, thereby potentially attenuating cognitive changes.

\section{Discussion}

Here we show that higher sTREM2 levels are associated with attenuated ApoE4-effects on future global cognitive and memory decline as well as AD-typical hippocampal neurodegeneration in a large sample of cognitively normal to AD dementia subjects. These effects were statistically independent of baseline A $\beta$-levels or diagnostic status. Importantly, CSF sTREM2 levels were - in contrast to CSF markers of $A \beta$ and tau pathology - not associated with ApoE4-carriage, confirming previous evidence that CSF sTREM2 concentrations [43] or PETassessed microglial activation [44] are per se unrelated to ApoE4-associated genetic AD risk. Given that CSF
sTREM2 levels most likely reflect the expression levels of signaling competent TREM2 on activated microglia $[27,30]$, our findings indicate that a higher TREM2related neuroimmune response may be protective against the clinical and neurodegenerative consequences of ApoE4-carriage, the strongest genetic risk factor for sporadic AD.

Consistent with previous studies showing associations between ApoE4 and more abnormal AD biomarkers, we found that ApoE4-carriers had overall stronger abnormal changes in CSF markers of $A \beta_{1-42}$ and p-tau ${ }_{181}$ at baseline, supporting the role of ApoE4 as a major AD risk factor $[45,46]$. In addition, ApoE4 carriers showed faster rates of cognitive decline and neurodegeneration, suggesting greater risk for developing AD-related cognitive impairment in ApoE4 positive individuals of the ADNI cohort. However, ApoE4-related risk for accelerated cognitive decline and neurodegeneration was attenuated at higher sTREM2 levels, independent of diagnosis or baseline levels of AD pathology, suggesting that relatively high microglial sTREM2 may compensate the ApoE4related increase in the risk to develop cognitive decline. Similarly, we showed previously in biomarker defined AD patients (i.e. patients with abnormal amyloid and tau levels), that higher sTREM2-levels were associated with delayed cognitive decline and clinical progression [34]. 
The current results critically extend these previous findings [34], suggesting that a TREM2-related neuroimmune response may attenuate the effects of the strongest known genetic risk factor for developing sporadic AD. These findings are of high clinical relevance since TREM2 signaling is potentially modifiable, as shown recently with TREM2-agonistic antibodies [26].

For neurodegeneration, we found that a higher TREM2-related microglia response attenuates the effect of ApoE4-carriage on atrophy of the hippocampus. Again, these results were independent of primary $A D$ pathology markers (i.e. CSF $A \beta_{1-42}$, p-tau 181 ). We and others have previously reported reduced grey matter atrophy at higher CSF sTREM2 levels at a given level of primary $\mathrm{AD}$ pathology in $\mathrm{AD}$ patients [47]. A study combining TSPO-PET with structural MRI in AD patients could show that higher TSPO-PET was associated with higher grey matter volume, favoring a neuroprotective effect of microglial activation [44]. The latter study further reported that higher TSPO-related grey matter volume was associated with better cognition, suggesting that higher grey matter volume indeed reflects preserved neuronal integrity, rather than inflammation-mediated edema [44]. In the context of the current findings, it is thus possible that a TREM2-related neuroimmune response in ApoE4-carriers attenuates either an amyloidinduced deposition of tau pathology itself or the downstream consequences of tau that ultimately lead to neurodegeneration and cognitive decline [48]. Together, these previous findings suggest that protective effects of TREM2 may be observed on different levels, e.g. on primary AD pathology itself or on its' consequences. It will thus be important for future studies to address these questions on different levels, e.g. by disentangling the molecular interactions between microglia and primary $\mathrm{AD}$ pathology and by combining PET-imaging of primary AD pathologies and microglial activation with MRI and CSF sTREM2 assessments to better understand the interplay of microglial activation with in vivo markers of $\mathrm{AD}$.

Whether a TREM2-related microglial response to $\mathrm{AD}$ pathology is adaptive or detrimental for the development of AD has been intensely debated with conflicting results from preclinical studies [49]. Supporting a protective role of TREM2, preclinical studies have reported that TREM2-related microglial activation supports phagocytosis of $A \beta$ [20], limits amyloid [22] and tau seeding [24] as well as tau hyperphosphorylation [23]. Supporting this, experimentally increasing sTREM2 levels in 5xFAD mice enhances microglial proliferation and phagocytosis of $A \beta$ and limits $A \beta$ neurotoxicity [50]. In agreement with this finding, we showed recently in humans and APP mice that elevated sTREM2 levels are associated with slower PET-assessed $A \beta$ accumulation [51]. In a similar vein, microglia modulation via TREM2 agonistic antibodies has been shown to improve $A \beta$ phagocytosis in transgenic AD-mouse models [26]. A protective effect of TREM2 is further suggested by post-mortem assessments, showing that TREM2 function promotes microglial clustering and $A \beta$ plaque encapsulation that is associated with attenuated accumulation of tau pathology [24]. Similarly, studies in pure tauopathy mice (i.e. P301S) have shown that TREM2 overexpression ameliorates tau hyperphosphorylation, neurodegeneration and cognitive decline, by suppressing neuroinflammation induced tau kinases [23, 25]. In contrast, others have argued that TREM2 exacerbates both amyloid and tau pathology in $\mathrm{AD}$ mice [52]. Conflicting findings from animal models may stem from various factors, including the use of different disease models or the investigation of TREM2 at different disease stages or severity levels of primary $\mathrm{AD}$ pathology [49]. Despite conflicting pre-clinical findings on a protective vs. detrimental role of TREM2, our current and previous findings in humans $[34,51]$ favor a protective role of TREM2 against the development of $\mathrm{AD}$ pathology, $\mathrm{AD}$ related cognitive decline and neurodegeneration.

Several caveats should be considered when interpreting the current results. First, while the link between ApoE4 and $\mathrm{AD}$ pathology is clearly established [46], there exist mixed and partly inconsistent reports on whether ApoE4carriage is indeed associated with cognition and cognitive changes, depending on age and selection of the study population $[8,9,11,53-55]$. However, results from several previous studies indicate that ApoE4-associated cognitive changes are pronounced specifically at older age [9-11]. Thus, we believe that the current sample with a mean age of $\sim 73$ years is well-suited to study ApoE4 effects on cognitive changes. Second, previous studies have reported increases of sTREM2 in AD, specifically in $A \beta$ and taupositive subjects [32,34]. Hence, the absence of groupspecific (i.e. $\mathrm{CN}, \mathrm{MCI}, \mathrm{AD}$ dementia) sTREM2 increases in the current study is potentially driven by our study design including subjects with mixed levels of both $A \beta$ and tau pathology across diagnostic groups. Third, CSF sTREM2 levels are only an indirect measure of TREM2 signaling [30], hence direct conclusions on the level of microglia activation cannot be drawn unless our findings are replicated with PET imaging of microglial activation or by TREM2 antibody mediated microglial modulation [26]. Fourth, microglial activation in neurodegenerative diseases is highly complex and requires an interplay of multiple signaling pathways besides TREM2 [56], which were not assessed in the current study. Future studies will thus need to investigate further how the complex molecular signature of activated microglia may modulate $\mathrm{AD}$ progression. Fifth, the current study is observational in nature, hence we emphasize that causative conclusions are not necessarily implied by our findings. 


\section{Conclusions}

In conclusion, we show that higher sTREM2 levels attenuate the effect of ApoE4-carriage, i.e. the strongest genetic risk factor for sporadic $\mathrm{AD}$, on future cognitive decline and neurodegeneration. These findings have important clinical implications, since TREM2 may reduce the overall risk to develop $\mathrm{AD}$. Together, our findings suggest that targeting TREM2 could serve as a promising strategy for treating and preventing AD.

\section{Supplementary information}

Supplementary information accompanies this paper at https://doi.org/10. 1186/s13024-020-00407-2.

\section{Additional file 1.}

\section{Abbreviations}

Aß: Amyloid-beta; AD: Alzheimer's disease; ADAS13: Alzheimer's disease assessment scale 13; ADAM10/17: A Disintegrin and metalloproteinase domain-containing protein 10/17; ADNI: Alzheimer's disease neuroimaging initiative; ANCOVA: Analysis of Covariance; ApoE4: Apolipoprotein 4; CDR: Clinical dementia rating; CSF: Cerebrospinal fluid; DAP12: DNAXactivating protein of 12 kDA; ELISA: Enzyme-linked immunosorbent assay; GWAS: Genome wide association studies; MMSE: Mini-Mental State Examination; MPRAGE: Magentization prepared rapid gradient echo; MRI: Magnetic Resonance Imaging; PET: Positron Emission Tomography; ptau: Phosphorylated tau; TREM2: Triggering receptor expressed on myeloid cells 2; TSPO: Translocator Protein; sTREM2: Soluble TREM2

\section{Acknowledgements}

Data used in preparation of this manuscript were obtained from the ADNI database (adni. loni.usc.edu). As such, the investigators within the ADNI study contributed to the design and implementation of ADNI and/or provided data but did not participate in analysis or writing of this paper. A complete listing of ADNI investigators can be found online at: http:// adni. loni.usc.edu/wp-content/uploads/how_to_apply/

ADNI_Acknowledgement_List.pdf.

All sTREM2 measurements in the CSF have been performed by the lab of C. Haass at the DZNE Munich, Germany.

\section{Authors' contributions}

$\mathrm{NF}, \mathrm{CH}, \mathrm{ME}$ : conception and design of the study, acquisition and analysis of data; drafting the manuscript MSC, LF, AM, TJH, EMR, BN, LS, JQT, GK: acquisition and analysis of data. MD: conception and design of the study. The authors read and approved the final manuscript.

\section{Funding}

The study was funded by grants from the LMUexcellent and Legerlotz Foundations (to ME), LMU intramural funds (FöFoLe, 1032, awarded to NF) and the Hertie foundation for clinical neurosciences (awarded to NF). This work was further supported by the Deutsche Forschungsgemeinschaft (DFG) within the framework of the Munich Cluster for Systems Neurology (EXC 2145 SyNergy), the DFG grant for major research instrumentation (DFG, INST 409/193-1 FUGG) and the Helmholtz-Gemeinschaft (Zukunftsthema "Immunology and Inflammation" (ZT-0027)). MSC received funding from the European Union's Horizon 2020 Research and Innovation Program under the Marie Sklodowska-Curie action grant agreement No 752310. MSC receives funding from Instituto de Salud Carlos III (PI19/00155). ADNI data collection and sharing for this project was funded by the ADNI (National Institutes of Health Grant U01 AG024904) and DOD ADNI (Department of Defense award number W81XWH-12-2-0012). ADNI is funded by the National Institute on Aging, the National Institute of Biomedical Imaging, and Bioengineering, and through contributions from the following: AbbVie, Alzheimer's Association; Alzheimer's Drug Discovery Foundation; Araclon Biotech; BioClinica, Inc:; Biogen; Bristol-Myers Squibb Company; CereSpir, Inc.; Cogstate; Eisai Inc.; Elan Phar- maceuticals, Inc.; Eli Lilly and Company; Eurolmmun; F. Hoffmann-La Roche Ltd. and its affiliated company Genentech, Inc.; Fujirebio; GE
Healthcare; IXICO Ltd.; Janssen Alzheimer Immunotherapy Research \& Development, LLC.; Johnson \& Johnson Phar- maceutical Research \& Development LLC.; Lumosity; Lundbeck; Merck \& Co., Inc.; Meso Scale Diagnostics, LLC.; NeuroRx Research; Neurotrack Technologies; Novartis Pharmaceuticals Corporation; Pfizer Inc.; Piramal Imaging; Servier; Takeda Pharma- ceutical Company; and Transition Therapeutics. The Canadian Institutes of Health Research is providing funds to support ADNI clinical sites in Canada. Private sector contributions are facilitated by the Foundation for the National Institutes of Health (www.fnih.org). Open Access funding enabled and organized by Projekt DEAL.

\section{Availability of data and materials}

The dataset supporting the conclusions of this manuscript is available at the ADNI website (http://adni.loni.usc.edu/).

Data used in preparation of this article were obtained from the Alzheimer's Disease Neuroimaging Initiative (ADNI) database (adni.loni.usc.edu). As such, the investigators within the ADNI contributed to the design and implementation of ADNI and/or provided data but did not participate in analysis or writing of this report. A complete listing of ADNI investigators can be found in the appendix ("ADNI_coinvestigators.docx").

Ethics approval and consent to participate

ADNI was ethically approved by the institutional review board of all participating sites, subjects provided written informed consent.

\section{Consent for publication}

Not applicable.

\section{Competing interests}

The authors declare that they have no competing interests.

\section{Author details}

${ }^{1}$ Institute for Stroke and Dementia Research (ISD), University Hospital, Ludwig Maximilian University (LMU), Munich, Germany. ${ }^{2}$ Barcelonaßeta Brain Research Center (BBRC), Pasqual Maragall Foundation, Barcelona, Spain. ${ }^{3}$ IMIM (Hospital del Mar Medical Research Institute), Barcelona, Spain. ${ }^{4}$ Servei de Neurologia, Hospital del Mar, Barcelona, Spain. ${ }^{5}$ Vanderbilt Memory \& Alzheimer's Center, Vanderbilt University Medical Center, Nashville, USA. ${ }^{6}$ Chair of Metabolic Biochemistry, Biomedical Center (BMC), Faculty of Medicine, Ludwig-Maximilians-Universität München, Munich, Germany. ${ }^{7}$ Center for Neurodegenerative Disease Research, Institute on Aging, Perelman School of Medicine University, Philadelphia, USA. ${ }^{8}$ Department of Pathology and Laboratory Medicine, Perelman School of Medicine, University of Pennsylvania, Philadelphia, PA, USA. ${ }^{9}$ Munich Cluster for Systems Neurology, Munich, Germany. ${ }^{10}$ German Center for Neurodegenerative Diseases (DZNE), Munich, Germany. ${ }^{11}$ ISAR Bioscience, Planegg, Germany.

Received: 6 August 2020 Accepted: 24 September 2020 Published online: 08 October 2020

\section{References}

1. Belloy ME, Napolioni V, Greicius MD. A quarter century of APOE and Alzheimer's disease: Progress to date and the path forward. Neuron. 2019; 101:820-38.

2. Qian J, Wolters FJ, Beiser A, Haan M, Ikram MA, Karlawish J, Langbaum JB, Neuhaus JM, Reiman EM, Roberts JS, et al. APOE-related risk of mild cognitive impairment and dementia for prevention trials: an analysis of four cohorts. PLoS Med. 2017:14:e1002254

3. Blacker D, Haines $J$, Rodes L, Terwedow H, Go RC, Harrell LE, Perry RT, Bassett SS, Chase G, Meyers D, et al. ApoE-4 and age at onset of Alzheimer's disease: the NIMH genetics initiative. Neurology. 1997;48:139-47.

4. Lautner R, Insel PS, Skillback T, Olsson B, Landen M, Frisoni GB, Herukka SK, Hampel $\mathrm{H}$, Wallin A, Minthon L, et al. Preclinical effects of APOE epsilon4 on cerebrospinal fluid Abeta42 concentrations. Alzheimers Res Ther. 2017;9:87.

5. Gonneaud J, Arenaza-Urquijo EM, Fouquet M, Perrotin A, Fradin S, de La Sayette V, Eustache F, Chetelat G. Relative effect of APOE epsilon4 on neuroimaging biomarker changes across the lifespan. Neurology. 2016;87: 1696-703.

6. Fleisher AS, Chen K, Liu X, Ayutyanont N, Roontiva A, Thiyyagura P, Protas H, Joshi AD, Sabbagh M, Sadowsky CH, et al. Apolipoprotein E epsilon4 and 
age effects on florbetapir positron emission tomography in healthy aging and Alzheimer disease. Neurobiol Aging. 2013;34:1-12.

7. Therriault J, Benedet AL, Pascoal TA, Mathotaarachchi S, Chamoun M, Savard M, Thomas E, Kang MS, Lussier F, Tissot C, et al. Association of Apolipoprotein E epsilon4 with medial temporal tau independent of amyloid-beta. JAMA Neurol. 2020;77:470-9.

8. Rawle MJ, Davis D, Bendayan R, Wong A, Kuh D, Richards M. Apolipoprotein-E (Apoe) epsilon4 and cognitive decline over the adult life course. Transl Psychiatry. 2018;8:18.

9. Caselli RJ, Dueck AC, Osborne D, Sabbagh MN, Connor DJ, Ahern GL, Baxter LC, Rapcsak SZ, Shi J, Woodruff BK, et al. Longitudinal modeling of age-related memory decline and the APOE epsilon4 effect. N Engl J Med. 2009;361:255-63.

10. Christensen H, Batterham PJ, Mackinnon AJ, Jorm AF, Mack HA, Mather KA, Anstey KJ, Sachdev PS, Easteal S. The association of APOE genotype and cognitive decline in interaction with risk factors in a 65-69 year old community sample. BMC Geriatr. 2008:8:14.

11. Makkar SR, Lipnicki DM, Crawford JD, Kochan NA, Castro-Costa E, Lima-Costa MF, Diniz BS, Brayne C, Stephan B, Matthews F, et al. APOE epsilon4 and the influence of sex, age, vascular risk factors, and ethnicity on cognitive decline. J Gerontol A Biol Sci Med Sci. 2020;75(10):1863-73. https://doi.org/ 10.1093/gerona/glaa116

12. Cacciaglia R, Molinuevo JL, Falcon C, Brugulat-Serrat A, Sanchez-Benavides G, Gramunt N, Esteller M, Moran S, Minguillon C, Fauria K, et al. Effects of APOE-epsilon4 allele load on brain morphology in a cohort of middle-aged healthy individuals with enriched genetic risk for Alzheimer's disease. Alzheimers Dement. 2018;14:902-12.

13. International Genomics of Alzheimer's Disease C. Convergent genetic and expression data implicate immunity in Alzheimer's disease. Alzheimers Dement. 2015;11:658-71

14. Jansen IE, Savage JE, Watanabe K, Bryois J, Williams DM, Steinberg S, Sealock J, Karlsson IK, Hagg S, Athanasiu L, et al. Genome-wide meta-analysis identifies new loci and functional pathways influencing Alzheimer's disease risk. Nat Genet. 2019;51:404-13.

15. Kunkle BW, Grenier-Boley B, Sims R, Bis JC, Damotte V, Naj AC, Boland A, Vronskaya M, van der Lee SJ, Amlie-Wolf A, et al. Genetic meta-analysis of diagnosed Alzheimer's disease identifies new risk loci and implicates Abeta, tau, immunity and lipid processing. Nat Genet. 2019;51:414-30.

16. Jonsson T, Stefansson H, Steinberg S, Jonsdottir I, Jonsson PV, Snaedal J, Bjornsson S, Huttenlocher J, Levey Al, Lah JJ, et al. Variant of TREM2 associated with the risk of Alzheimer's disease. N Engl J Med. 2013;368:107-16.

17. Mazaheri F, Snaidero N, Kleinberger G, Madore C, Daria A, Werner G, Krasemann S, Capell A, Trumbach D, Wurst W, et al. TREM2 deficiency impairs chemotaxis and microglial responses to neuronal injury. EMBO Rep. 2017;18:1186-98.

18. Takahashi K, Rochford CD, Neumann H. Clearance of apoptotic neurons without inflammation by microglial triggering receptor expressed on myeloid cells-2. J Exp Med. 2005;201:647-57.

19. Kleinberger $G$, Yamanishi $Y$, Suarez-Calvet M, Czirr E, Lohmann E, Cuyvers $E_{\text {, }}$ Struyfs $\mathrm{H}$, Pettkus N, Wenninger-Weinzierl A, Mazaheri F, et al. TREM2 mutations implicated in neurodegeneration impair cell surface transport and phagocytosis. Sci Transl Med. 2014;6:243ra286.

20. Yeh FL, Wang Y, Tom I, Gonzalez LC, Sheng M. TREM2 binds to Apolipoproteins, including APOE and CLU/APOJ, and thereby facilitates uptake of amyloid-Beta by microglia. Neuron. 25;75(10):1863-1873. https:// doi.org/10.1093/gerona/glaa116.

21. Wang Y, Cella M, Mallinson K, Ulrich JD, Young KL, Robinette ML, Gilfillan S, Krishnan GM, Sudhakar S, Zinselmeyer BH, et al. TREM2 lipid sensing sustains the microglial response in an Alzheimer's disease model. Cell. 2015;160:1061-71.

22. Parhizkar S, Arzberger T, Brendel M, Kleinberger G, Deussing M, Focke C, Nuscher B, Xiong M, Ghasemigharagoz A, Katzmarski N, et al. Loss of TREM2 function increases amyloid seeding but reduces plaque-associated ApoE. Nat Neurosci. 2019;22:191-204.

23. Jiang T, Zhang YD, Gao Q, Ou Z, Gong PY, Shi JQ, Wu L, Zhou JS. TREM2 ameliorates neuronal tau pathology through suppression of microglial inflammatory response. Inflammation. 2018;41:811-23.

24. Leyns CEG, Gratuze M, Narasimhan S, Jain N, Koscal LJ, Jiang H, Manis M, Colonna M, Lee VMY, Ulrich JD, Holtzman DM. TREM2 function impedes tau seeding in neuritic plaques. Nat Neurosci. 2019;22:1217-22.

25. Jiang T, Zhang YD, Chen Q, Gao Q, Zhu XC, Zhou JS, Shi JQ, Lu H, Tan L, Yu JT. TREM2 modifies microglial phenotype and provides neuroprotection in P301S tau transgenic mice. Neuropharmacology. 2016;105:196-206.
26. Schlepckow K, Monroe KM, Kleinberger G, Cantuti-Castelvetri L, Parhizkar S, Xia D, Willem M, Werner G, Pettkus N, Brunner B, et al. Enhancing protective microglial activities with a dual function TREM2 antibody to the stalk region. EMBO Mol Med. 2020;12:e11227.

27. Suarez-Calvet $M$, Kleinberger $G$, Araque Caballero MA, Brendel $M$, Rominger A, Alcolea D, Fortea J, Lleo A, Blesa R, Gispert JD, et al. sTREM2 cerebrospinal fluid levels are a potential biomarker for microglia activity in early-stage Alzheimer's disease and associate with neuronal injury markers. EMBO Mol Med. 2016:8:466-76.

28. Heslegrave A, Heywood W, Paterson R, Magdalinou N, Svensson J, Johansson P, Ohrfelt A, Blennow K, Hardy J, Schott J, et al. Increased cerebrospinal fluid soluble TREM2 concentration in Alzheimer's disease. Mol Neurodegener. 2016;11:3.

29. Piccio L, Deming Y, Del-Aguila JL, Ghezzi L, Holtzman DM, Fagan AM, Fenoglio C, Galimberti D, Borroni B, Cruchaga C. Cerebrospinal fluid soluble TREM2 is higher in Alzheimer disease and associated with mutation status. Acta Neuropathol. 2016;131:925-33.

30. Brendel M, Kleinberger G, Probst F, Jaworska A, Overhoff F, Blume T, Albert $\mathrm{NL}$, Carlsen J, Lindner S, Gildehaus FJ, et al. Increase of TREM2 during aging of an Alzheimer's disease mouse model is paralleled by microglial activation and amyloidosis. Front Aging Neurosci. 2017;9:8

31. Kleinberger G, Brendel M, Mracsko E, Wefers B, Groeneweg L, Xiang X, Focke C, Deussing M, Suarez-Calvet M, Mazaheri F, et al. The FTD-like syndrome causing TREM2 T66M mutation impairs microglia function, brain perfusion, and glucose metabolism. EMBO J. 2017;36:1837-53.

32. Suarez-Calvet M, Morenas-Rodriguez E, Kleinberger G, Schlepckow K, Araque Caballero MA, Franzmeier N, Capell A, Fellerer K, Nuscher B, Eren E, et al. Early increase of CSF sTREM2 in Alzheimer's disease is associated with tau related-neurodegeneration but not with amyloid-beta pathology. Mol Neurodegener. 2019;14:1.

33. Suarez-Calvet M, Araque Caballero MA, Kleinberger G, Bateman RJ, Fagan AM, Morris JC, Levin J, Danek A, Ewers M, Haass C, Dominantly Inherited Alzheimer N, et al. Sci Transl Med. 2016;8:369ra178.

34. Ewers M, Franzmeier N, Suarez-Calvet M, Morenas-Rodriguez E, Caballero MAA, Kleinberger G, Piccio L, Cruchaga C, Deming Y, Dichgans M, et al. Increased soluble TREM2 in cerebrospinal fluid is associated with reduced cognitive and clinical decline in Alzheimer's disease. Sci Transl Med. 2019;11(507):eaav6221. https://doi.org/10.1126/scitranslmed.aav6221..

35. Petersen RC, Aisen PS, Beckett LA, Donohue MC, Gamst AC, Harvey DJ, Jack CR Jr, Jagust WJ, Shaw LM, Toga AW, et al. Alzheimer's disease neuroimaging initiative (ADNI): clinical characterization. Neurology. 2010;74: 201-9.

36. Hansson O, Seibyl J, Stomrud E, Zetterberg H, Trojanowski JQ, Bittner T, Lifke V, Corradini V, Eichenlaub U, Batrla R, et al. CSF biomarkers of Alzheimer's disease concord with amyloid-beta PET and predict clinical progression: a study of fully automated immunoassays in BioFINDER and ADNI cohorts. Alzheimers Dement. 2018;14:1470-81.

37. Tosun D, Schuff N, Shaw LM, Trojanowski JQ, Weiner MW, Alzheimer's disease Neurolmaging I. Relationship between CSF biomarkers of Alzheimer's disease and rates of regional cortical thinning in ADNI data. J Alzheimers Dis. 2011;26(Suppl 3):77-90.

38. Jack CR Jr, Barnes J, Bernstein MA, Borowski BJ, Brewer J, Clegg S, Dale AM, Carmichael O, Ching C, DeCarli C, et al. Magnetic resonance imaging in Alzheimer's disease neuroimaging initiative 2. Alzheimers Dement. 2015;11: 740-56.

39. Crane PK, Carle A, Gibbons LE, Insel P, Mackin RS, Gross A, Jones RN, Mukherjee S, Curtis SM, Harvey D, et al. Development and assessment of a composite score for memory in the Alzheimer's disease neuroimaging initiative (ADNI). Brain Imaging Behav. 2012;6:502-16.

40. Mohs RC, Knopman D, Petersen RC, Ferris SH, Ernesto C, Grundman M, Sano M, Bieliauskas L, Geldmacher D, Clark C, Thal LJ. Development of cognitive instruments for use in clinical trials of antidementia drugs: additions to the Alzheimer's Disease Assessment Scale that broaden its scope. The Alzheimer's Disease Cooperative Study. Alzheimer Dis Assoc Disord. 1997; 11 (Suppl 2):S13-21.

41. Rosen WG, Mohs RC, Davis KL. A new rating scale for Alzheimer's disease. Am J Psychiatry. 1984;141:1356-64.

42. Preische O, Schultz SA, Apel A, Kuhle J, Kaeser SA, Barro C, Graber S, KuderBuletta E, LaFougere C, Laske C, et al. Serum neurofilament dynamics predicts neurodegeneration and clinical progression in presymptomatic Alzheimer's disease. Nat Med. 2019;25:277-83. 
43. Gispert JD, Monte GC, Suarez-Calvet M, Falcon C, Tucholka A, Rojas S, Rami L, Sanchez-Valle R, Llado A, Kleinberger G, et al. The APOE epsilon4 genotype modulates CSF YKL-40 levels and their structural brain correlates in the continuum of Alzheimer's disease but not those of sTREM2. Alzheimers Dement (Amst). 2017;6:50-9.

44. Hamelin L, Lagarde J, Dorothee G, Leroy C, Labit M, Comley RA, de Souza LC, Corne H, Dauphinot L, Bertoux M, et al. Early and protective microglial activation in Alzheimer's disease: a prospective study using 18F-DPA-714 PET imaging. Brain. 2016;139:1252-64.

45. Risacher SL, Kim S, Nho K, Foroud T, Shen L, Petersen RC, Jack CR Jr, Beckett LA, Aisen PS, Koeppe RA, et al. APOE effect on Alzheimer's disease biomarkers in older adults with significant memory concern. Alzheimers Dement. 2015;11:1417-29.

46. Liu Y, Yu JT, Wang HF, Han PR, Tan CC, Wang C, Meng XF, Risacher SL, Saykin AJ, Tan L. APOE genotype and neuroimaging markers of Alzheimer's disease: systematic review and meta-analysis. J Neurol Neurosurg Psychiatry. 2015;86:127-34

47. Gispert JD, Suarez-Calvet M, Monte GC, Tucholka A, Falcon C, Rojas S, Rami L, Sanchez-Valle R, Llado A, Kleinberger G, et al. Cerebrospinal fluid sTREM2 levels are associated with gray matter volume increases and reduced diffusivity in early Alzheimer's disease. Alzheimers Dement. 2016;12:1259-72.

48. La Joie R, Visani AV, Baker SL, Brown JA, Bourakova V, Cha J, Chaudhary K, Edwards $L$, laccarino $L$, Janabi $M$, et al. Prospective longitudinal atrophy in Alzheimer's disease correlates with the intensity and topography of baseline tau-PET. Sci Transl Med. 2020;12.

49. Gratuze M, Leyns CEG, Holtzman DM. New insights into the role of TREM2 in Alzheimer's disease. Mol Neurodegener. 2018;13:66.

50. Zhong L, Xu Y, Zhuo R, Wang T, Wang K, Huang R, Wang D, Gao Y, Zhu Y, Sheng $X$, et al. Soluble TREM2 ameliorates pathological phenotypes by modulating microglial functions in an Alzheimer's disease model. Nat Commun. 2019;10:1365.

51. Ewers M, Biechele G, Suarez-Calvet M, Sacher C, Blume T, MorenasRodriguez E, Deming Y, Piccio L, Cruchaga C, Kleinberger G, et al. Higher CSF sTREM2 and microglia activation are associated with slower rates of beta-amyloid accumulation. EMBO Mol Med. 2020;12:e12308.

52. Jay TR, Miller CM, Cheng PJ, Graham LC, Bemiller S, Broihier ML, Xu G, Margevicius D, Karlo JC, Sousa GL, et al. TREM2 deficiency eliminates TREM2+ inflammatory macrophages and ameliorates pathology in Alzheimer's disease mouse models. J Exp Med. 2015;212:287-95.

53. Bunce D, Bielak AA, Anstey KJ, Cherbuin N, Batterham PJ, Easteal S. APOE genotype and cognitive change in young, middle-aged, and older adults living in the community. J Gerontol A Biol Sci Med Sci. 2014;69:379-86.

54. Growdon JH, Locascio JJ, Corkin S, Gomez-Isla T, Hyman BT. Apolipoprotein E genotype does not influence rates of cognitive decline in Alzheimer's disease. Neurology. 1996;47:444-8.

55. Jorm AF, Mather KA, Butterworth P, Anstey KJ, Christensen H, Easteal S. APOE genotype and cognitive functioning in a large age-stratified population sample. Neuropsychology. 2007;21:1-8.

56. Krasemann S, Madore C, Cialic R, Baufeld C, Calcagno N, El Fatimy R, Beckers L, O'Loughlin E, Xu Y, Fanek Z, et al. The TREM2-APOE pathway drives the transcriptional phenotype of dysfunctional microglia in neurodegenerative diseases. Immunity. 2017;47:566-81 e569.

\section{Publisher's Note}

Springer Nature remains neutral with regard to jurisdictional claims in published maps and institutional affiliations.

Ready to submit your research? Choose BMC and benefit from:

- fast, convenient online submission

- thorough peer review by experienced researchers in your field

- rapid publication on acceptance

- support for research data, including large and complex data types

- gold Open Access which fosters wider collaboration and increased citations

- maximum visibility for your research: over $100 \mathrm{M}$ website views per year

At BMC, research is always in progress.

Learn more biomedcentral.com/submissions 